\section{P855 MULTI-PEPTIDE ELISAS OVERCOME CROSS-REACTIVITY AND INADEQUATE SENSITIVITY OF CHLAMYDIA TRACHOMATIS AND C. PNEUMONIAE SEROLOGY}

Kh Shamsur Rahman, Bernhard Kaltenboeck*. Auburn University, Pathobiology, Auburn, USA

\subsection{6/sextrans-2019-sti.897}

Background Chlamydia spp. serology is compromised by crossreactivity of classical antigens. For specific detection of antitrachomatis (Ctr) and anti-C. pneumoniae (Cpn) antibodies, we developed and validated novel peptide ELISAs.

Methods Strongly reactive peptide antigens of 24 Ctr- and 48 Cpn-specific B-cell epitopes of multiple immunodominant chlamydial proteins were used in this study. For specific detection of anti-Ctr and anti-Cpn antibodies, 185 human sera were tested in colorimetric ELISAs with mixtures of 12-24 Ctr or Cpn peptide antigens using polyclonal anti-human IgG-HRP conjugates. For comparative evaluation, these sera were tested with 4 Ctr and 4 Cpn commercial IgG ELISAs.

Results In commercial ELISAs, Ctr and Cpn individual serum reactivity was $54 \%$ biased towards positivity for both species (co-positivity), but unbiased in Ctr and Cpn peptide antibody assays. This finding suggested a severe specificity problem (cross-reactivity) of commercial ELISAs, but not peptide assays. Using hyperimmune mouse sera against each of 11 Chlamydia spp., we confirmed that commercial Ctr and Cpn ELISA antigens are cross-reactive among all Chlamydia spp., but Cpn and Ctr peptide antigens react specifically only with antisera against the cognate chlamydial species. By comparison at $90 \%$ specificity to a Ctr-peptide composite reference standard (CRS) for human anti-Ctr antibody status, the Ctr mixed peptide assays showed $86-83 \%$ sensitivity, significantly higher than the 59-34\% sensitivity of 4 commercial anti-Ctr ELISAs. Relative to a Cpn-peptide CRS, the Cpn mixed peptide assay showed $86-80 \%$ sensitivity at $90 \%$ specificity, significantly higher than the $48-25 \%$ sensitivity of 4 commercial anti-Cpn ELISAs.

Conclusion For detection of anti-Ctr and -Cpn antibodies, commercial ELISAs are not suitable due to cross-reactivity. In contrast, mixed peptide assays are accurate with simultaneous high specificity and sensitivity, and reliably determine anti-Ctr and anti-Cpn antibody prevalence. With convenient use for non-specialized laboratories, these peptide ELISAs will improve Ctr and Cpn serodiagnosis.

Disclosure No significant relationships.

\section{P856 PRIVACY AND TECHNOLOGY FOR YOUNG BLACK MEN WHO HAVE SEX WITH MEN IN THE SOUTHERN US RELATED TO SEXUAL CARE AND RESEARCH}

${ }^{1}$ Ellen Eaton, ${ }^{1}$ Christina Muzny*, ${ }^{2}$ Eric Ford. ' University of Alabama at Birmingham, Medicine, Birmingham, USA; ${ }^{2}$ Ryals School of Public Health, Health Care Organization and Policy, Birmingham, USA

\subsection{6/sextrans-2019-sti.898}

Background Technology, such as text messaging and mobile apps, has been integrated into health care. Technology preferences of underrepresented young black men who have sex with men (YB MSM) remain unknown but likely influence the use of health care services. The objective was to query the preferences of YB MSM related to technology regarding sexual health care and research. We hypothesized that YB MSM would prefer the use of social media and mobile applications (apps).

Methods We recruited YB MSM in Birmingham, Alabama to participate in a discrete choice analysis of sexual health preferences. Participants were given a link to an electronic survey, which queried their sociodemographic status and preferences for STI testing and research. We specifically queried the use of technology to notify of STI results and recruit for research in addition to privacy concerns.

Results 33 YB MSM met criteria: median age was 28, most were homosexual $(n=27,82 \%)$ and the remaining bisexual, $17(52 \%)$ had HIV, 24 (73\%) reported a prior STI, $16(48 \%)$ live below the state federal poverty level, and 11 (33\%) were uninsured. When asked, the best and worst way to be contacted with STI testing results, 21/33 (64\%) preferred phone notification. Further, (52\%) and (39\%) said texting and email were the worst option, respectively. When asked the best way to recruit YB MSM for sexual health research, 16/33 (48\%) reported social media. The most frequently recommended social media sites were Facebook (33\%) and Instagram (27\%). When asked their greatest concern about STI testing, 8/33 (24\%) reported privacy concerns. None expressed concerns about limited access to technology.

Conclusion YB MSM in the Southern U.S. prefer social media and mobile apps for research recruitment but not for communication about personal health. When it comes to STI testing, privacy remains a significant concern for YB MSM.

Disclosure No significant relationships.

\section{P857 PENILE MICROBIOME AND URINARY CYTOKINES OF KENYAN MEN WHO HAVE SEX WITH MEN AND MEN WHO HAVE SEX WITH WOMEN}

${ }^{1}$ Supriya Mehta*, 'Debarghya Nandi, ${ }^{2}$ Duncan Okal, ${ }^{2}$ George N'Gety, ${ }^{3}$ Stefan Green, ${ }^{2}$ Fredrick Otieno, ${ }^{2}$ Eve Obondi, ${ }^{4}$ Jennifer Kinslow, ${ }^{1}$ Dulal Bhaumik, ${ }^{3}$ Robert Bailey, ${ }^{4}$ Alan Landay. ${ }^{1}$ University of Illinois at Chicago, Epidemiology and Biostatistics, Chicago, USA; ${ }^{2}$ Nyanza Reproductive Health Society, Kisumu, Kenya; ${ }^{3}$ University of Illinois at Chicago, Chicago, USA; ${ }^{4}$ Rush University, Chicago, USA

\subsection{6/sextrans-2019-sti.899}

Background MSM are disproportionately affected by HIV and STIs compared to men who have sex with women (MSWomen). This may be due in part to different burden of mucosal inflammation. We compared penile microbial composition between MSM and MSWomen and association with mucosal inflammation.

Methods In this cross-sectional study, we enrolled 43 MSM and 43 MSWomen, who were HIV negative and matched on age and circumcision status. The penile microbiome was assessed via meatal swab, with $16 \mathrm{~S}$ rRNA gene amplicon sequencing. Urinary cytokine concentrations (TNF- $\alpha / \mathrm{IL}-1 \beta / \mathrm{IL}$ 8/IL-10/IP-10) were measured using Luminex. Random Forest (RF) identified genus-level taxa differing between MSM and MSWomen. Taxa from RF were regressed on cytokine outcomes, with multiple testing correction and information criterion model selection.

Results Men were median age 24 and 77\% circumcised. There were substantial differences in educational attainment, employment, alcohol and drug use, condom use, and number of sexual partners, with MSM having greater behavioral risks. Microbiome composition differed markedly between MSM and MSWomen: RF discriminated between MSM and MSWomen with $84 \%$ accuracy. Taxa with greatest 